\title{
Warum ist das vierte Evangelium fur ein Werk des Apostels Johannes erklärt worden?
}

\author{
Von P. Corssen in Berlin.
}

I.

Die Presbyter des Irenaeus.

Das vierte Evangelium hat, soweit wir es mit Sicherheit zurückverfolgen können, für ein Werk des Apostels Johannes gegolten. Auch der Widerspruch, der im zweiten Jahrhundert gegen das Evangelium erhoben wurde, bestätigt dies nur. Denn die sogenannten Aloger hielten es wohl für eine Fälschung, aber für eine Fälschung auf den Namen des Johannes. „Das Evangelium auf den Namen des Johannes, sagten sie, lügt" (Epiphanius LI, I8). Indem sie zu beweisen suchten, dass es von Johannes nicht herrühren könne, sprachen sie aus, dass die allgemeine Meinung Johannes für den Verfasser halte. Diese Thatsache ist keineswegs so selbstverständlich, wie sie unserer Gewohnheit erscheint. Denn wenn zwar in dem Schlusskapitel des Evangeliums der Lieblingsschüler ausdrücklich für den Verfasser erklärt wird, so ist doch für uns nicht unmittelbar einleuchtend, warum in diesem Lieblingsschüler eben der Apostel Johannes zu erkennen sei. So vielfach auch die Frage behandelt ist, ob der Apostel Johannes der Verfasser des vierten Evangeliums sein kann, so scheint mir doch die Frage, wie man dazu gekommen ist, ihn dafür zu halten, noch nicht mit methodischer Gründlichkeit behandelt zu sein. Wie aber diese Frage für die Verfasserfrage überhaupt offenbar nicht ohne Bedeutung ist, so hat sie doch den Vorteil, nicht auf dem heissen Boden des eigentlichen Kampfplatzes zu liegen. Denn es handelt sich nicht um das Wesen des Evangeliums, sondern um die Entstehung einer Meinung, deren innere Berechtigung ausserhalb der Erörterung bleibt. 
Ich untersuche zunächst, ob die Kirche eine von dem Evangelium unabhängige Tradition über seinen Verfasser besessen hat oder besessen haben kann.

Wir finden eine solche Tradition, die in modificierter Form von der katholischen Kirche festgehalten ist und auch in evangelischen Kreisen viele Anhänger hat, bei dem Muratorischen Fragmentisten '. Er berichtet: „Johanneś, einer der Schüler, erklärte auf die Aufforderung seiner Mitschüler und -bischöfe: fastet mit mir (von) heute drei Tage und was einem jeden offenbart wird, lasst uns einander erzählen. In derselben Nacht wurde Andreas, einem der Apostel, offenbart, es solle unter Verantwortung aller Johannes in seinem Namen alles niederschreiben."

Aber der anonyme Fragmentist giebt diese Nachricht ohne alle Gewähr, woher er sie empfangen, und der jedenfalls ältere Irenaeus weiss oder sagt wenigstens nichts von ihr. Er beschränkt sich auf die Erklärung, dass das Evangelium von Johannes, dem Schüler des Herrn, in Ephesus zur Bekämpfung des Kerinth und der Nikolaiten geschrieben sei (III, I, I und II, I).

Hat es aber irgend eine Tradition darüber gegeben, dass und warum der Jünger Johannes das Evangelium geschrieben habe, so muss sie in den Kreisen der Presbyter gesucht werden, die nach Irenaeus' Behauptung den Apostel Johannes persönlich gekannt haben.

Über die Presbyter bei Irenaeus ist viel verhandelt worden, aber doch nicht so, dass daraus Gewinn für unsere Frage gezogen wäre oder ohne weiteres gezogen werden könnte. Eine bedeutende Förderung hat ihre Behandlung durch Harnacks gründliche und scharfsinnige Untersuchung in der Chronologie der altchr. Litt. S. $333 \mathrm{ff}$. gefunden, nachdem vorher besonders Lightfoot einige wesentliche Thatsachen ins Licht gestellt hatte.

Mit Recht tadeit Harnack an den Untersuchungen $Z$ ahns und anderer, dass sie zwischen den verschiedenen Presbytern, die von Irenaeus erwähnt werden, nicht unterscheiden. Siebenmal citiert er einen Presbyter, den er nach seiner eigenen Angabe selbst gehört hatte (IV, c. 27-32). Dieser hat nichts zu thun mit den Presbytern, auf die er sich als eine Mehrheit beruft. Auf diese allein aber kommt es hier an.

Fünfmal begegnen sie uns (II, 22, 5. V, 5, I. 30, I. 33, 3. 36, I und 2),

I Der vortreffliche englische Theologe Lightfoot sagt Contemporary Review, Bd. 26, S. 837 Anm.: Es hat mir immer geschienen, dass das Muratorische Fragment einen im wesentlichen zuverlässigen Bericht von den Vorgängen enthält, unter denen das vierte Evangelium entstanden ist. 
an Stcllen, die unter einander in engem Zusammenhang stehen. Dreimal wird hervorgehoben, dass sic Johannes persönlich gekannt haben (II, 22, 5. V, 30, I. 33, 3), der zweimal Schüler des Herrn genannt wird. An einer Stelle (II, 22, 5) heisst es, dass einige von ihnen auch andere Apostel gekannt hätten, und zweimal werden sie schlechthin Apostelschüler genannt. Jedesmal werden ihre Aussprüche im Praesens angeführt. Es ist mir nicht verständlich, wie ein Mann wie Jülicher dies so erklären will, als habe Irenaeus gemeint, jene Presbyter wären noch am Leben gewesen, als er dieses schrieb (Gött. gel. Anz. 1896, S. 847). Schon Lightfoot ${ }^{2}$ hat aus dem Praesens geschlossen, dass Irenaeus an diesen Stellen aus einer schriftlichen Quelle geschöpft hat, und den Weg zu dieser Quelle aufgezeigt, den Harnack dann noch genauer verfolgt hat.

Will man den Beweis der Abhängigkeit des Irenaeus führen, so geht man am besten von V, 33, 3 f. aus. Hier wird berichtet, was die Presbyter über die Zustände in dem tausendjährigen Reiche aus dem Munde des Apostels gehört haben wollen. „Die'Presbyter", heisst es, „die Johannes, den Schüler des Herrn, gesehen haben, erinnern sich von ihm gehört $z u$ haben, wie der Herr sie über jene Zeiten belehrte und sagte." Und nun wird die wunderbare Fruchtbarkeit, die dann auf Erden herrschen werde, geschildert. „Dies aber“, fährt Irenaeus fort, „bezeugt auch Papias, der Hörer des Johannes und Gefährte Polykarps, ein altehrwürdiger Mann, schriftlich in dem vierten seiner Bücher." Das klingt ja freilich so, als wolle Irenaeus das Zeugnis des Papias neben das der Presbyter stellen, besonders da er ihn einen Hörer des Johannes nennt. Aber wenn Irenaeus sagen wollte, Papias berichte an jenem Orte Selbstgehörtes, warum unterschied er ihn dann von den Presbytern, die den Johannes gesehen hatten? Oder wollte er, der sein Werk vor 173 nicht begonnen haben kann und vor I8I wahrscheinlich ' nicht begonnen hat (Harnack, Chronol. S. 320), zu verstehen geben, dass jene ungenannten Presbyter noch im Fleische wandelten und von jedermann befragt werden könnten? Aber in Wahrheit war Papias, wie schon Eusebius bemerkt, gar nicht .der Hörer des Johannes gewesen; vielmehr hatte er in der Vorrede seines Werkes ausdrücklich erklärt, dass er alles was er von Johannes und anderen Aposteln wusste, von anderen erkundet hatte. Wenn also Papias dasselbe erzählte, was Irenaeus nach den Presbytern berichtet, so konnte seine Erzählung schlechterdings auf niemand anders zurückgehen als auf eben diese Presbyter. Da es nun

I Contemporary Review, Bd. 26, p. 841 f. 
ausgeschlossen ist, dass die Presbyter zu Irenaeus' Zeit noch gelebt haben, er aber ihre Zeugnisse in derselben Weise wie Schriftworte als gegenwärtig behandelt, so müssen doch wohl diese, sei es an einem, sei es an verschiedenen Orten, schriftlich niedergelegt gewesen sein; und es dürfte doch wohl wahrscheinlich sein, dass mindestens in diesem einen Falle ihr Zeugnis sich bei Papias fand. Zur Gewissheit wird dies durch folgende Erwägung erhoben. Nachdem Irenaeus sich auf Papias berufen hat, geht es weiter: „Und er setzte hinzu und sagte: dies aber ist glaublich den Gläubigen. Und da Judas, sagte er, der Verräter es nicht glaubte und fragte: wie werden denn solche Erzeugnisse von Gott ${ }^{3}$ hervorgebracht werden? habe der Herr gesagt: Es werden es sehen, die dahin kommen werden". Hier zeigt sich deutlich, dass Irenaeus eine schriftliche Quelle citiert, die er verkürzt. Wer setzte denn hinzu und sagte? Dies schliesst durchaus nicht an das Vorhergehende an, denn da spricht der Herr in eigener Person. Es muss diese Rede des Herrn in der Quelle etwa so eingeleitet gewesen sein: „Johannes erzählte uns, wie der Herr die Jünger über das kommende Reich belehrte." Dann ist es klar, dass Johannes es war, der die Bemerkung hinzusetzte, mit der er den Übergang zu dem ungläubigen Judas gewann².

Irenaeus hat aber schwerlich selbst etwas anderes sagen wollen, als dieses: das Zeugnis der Presbyter ist ein mündliches, es existiert aber auch schriftlich, da Papias es aufgeschrieben hat. So bezeugt denn auch Papias die Geschichte.

Ist aber diese Erzählung aus Papias entlehnt, so sind die übrigen Zeugnisse der Presbyter, bei denen Papias' Name nicht erwähnt ist, auch auf ihn zurückzuführen. Denn alle in Betracht kommenden Stellen stehen, wie bereits bemerkt, unter sich in engem Zusammenhange. Sie handeln alle, bis auf eine (II, 22, 5), von zukünftigen Dingen und überall sind offenbar die Presbyter, die dafür zu Zeugen gemacht werden, dieselben, nämlich die, die den Johannes noch gekannt haben. $\mathrm{Da}$ sie als solche auch II, 22, 5 bezeichnet werden, so ist diese -Stelle mit den andern zu verbinden.

1 Die Ausgaben haben a Domino.

2 Es ist vermutet worden, dass Papias an dieser Stelle auch von Hippolyt, Comment. in Dan IV, 60, benutzt worden sei (s. Preuschen, Antilegom. S. 63). Aber Hippolyt lässt den Judas fragen: Und wer wird denn das sehen? und den Herrn antworten: Das werden die sehen, die es verdienen. Da Hippolyt die Reden direkt anführt, so ist nicht anzunehmen, dass er ungenau citiert. Ich glaube, dass Fippolyt eine andere Stelle des Papias vor Augen hatte und dass sie auch noch nachweisbar ist, aber der Nachweis würde hier zu weit fübren. 
Wir müssen uns also von Irenaeus an Papias wenden, wenn wir die Tradition über Johannes weiter verfolgen wollen. Dies nötigt uns, die schon bcrührte, vielbesprochene Stelle cingehender 7.4 behandeln, an der sich Papias über scin Verhältnis zu den Presbytern ausspricht.

Papias crklärte nach Eusebius h. e. III, 39 in seiner Vorrede: "Ich werde mich aber nicht bedenken, auch alles was ich einst von den

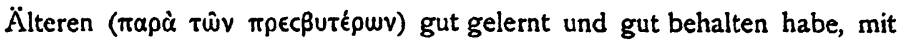

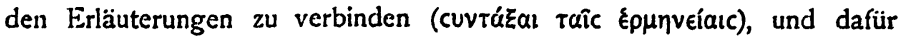
übernehme ich die Garantie. Denn ich hatte nicht, wie die meisten, meine Freude an dencn, die vieles sagen, sondern an denen, die Wahres lehren, und nicht an denen, die die fremden Gebote überliefern, sondern die von dem Herrn dem Glauben gegebenen und von der Wahrheit selbst stammenden. Wenn aber auch irgend einer kam, der in dem Gefolge der Älteren gewesen war, so pflegte ich nach den Worten der Älteren zu forschen, was Andreas oder was Petrus sagte oder was Philippus oder was Thomas oder Jacobus oder was Johannes oder Matthäus oder irgend ein anderer der Schüler des Herrn, und was Aristion und der Presbyter Johannes, die Schüler des Herrn, sagen. Denn ich glaubte nicht, dass das, was aus den Büchern kommt, mich so sehr fördere, wie

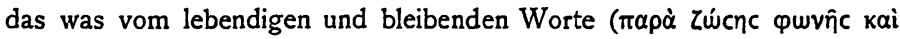
$\mu \in$ oúcnc) stammt".

Wenn Papias sagt, er habe seine Kunde von den Älteren, so klingt das, als habe er sie unmittelbar aus ihrem Munde. Aber die nähere Erklärung, die nachfolgt, zeigt, dass sie ihm nur mittelbar zugekommen war, durch Leute, die jene gehört hatten. Drückt sich Papias über das Verhältnis seiner Gewährsleute zu den Presbytern unzweideutig aus, so kann man dagegen zweifeln, wen er denn unter diesen Preşbytern versteht. Man könnte meinen, es seien eben die Jünger, Andreas, Petrus u.s.w., indem der Satz, was Andreas oder was Petrus sagte, den Ausdruck, die Worte der Älteren, aufnähme und erläutere. Aber das ist doch nicht wahrscheinlich, da diese durch die Bezeichnung, Schüler des Herrn, vielmehr von den Presbytern unterschieden zu werden scheinen. Ganz unmöglich aber wird die Annahme, wenn wir bedenken, dass in dem Werke selbst von den Presbytern die Rede war, die den Johannes und andere Apostel gesehen hatten. Da Papias in der Vorrede erklärt, dass er seine Nachrichten nicht von den Presbytern selbst bezogen habe, und da er in seinem Werke zwischen Presbytern und Aposteln unterscheidet, so gewinnen wir drei Stufen: Apostel, Presbyter und Nachfolger der Presbyter. Diese letzteren sind seine Gewährsleute. Folglich ist 
Papias erst im dritten Grade Zeuge von Aussprüchen und Erzählungen der Apostel.

Damit ist ausgeschlossen, dass Papias selbst ein Hörer des Apostels Johannes gewesen wäre, wie Irenaeus behauptet. Schon Eusebius hat bemerkt, dass diese Behauptung im Widerspruch mit dem Prooemium des Papias steht. Hätte er den Apostel Johannes noch gekannt, so

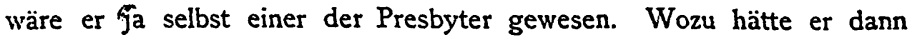
bei andern nach den Aussagen der Presbyter zu forschen brauchen? Warum nannte Irenaeus ihn dann nicht unter denen, die mit Johannes bis zu den Tagen Trajans zusammen waren? Warum beruft sich Irenaeus vielmehr nur auf das Zeugnis namenloser Presbyter statt auf den wohlbekannten Papias, der, wenn er wirklich Hörer des Johannes gewesen wäre, doch wohl mehr als eine einzige Geschichte von ihm zu erzählen gehabt hätte? Warum aber endlich unterscheidet er ihn an der einzigen Stelle, an der er sich auf ihn als auf den Hörer des Johannes beruft, von den Presbytern, die den Schüler des Herrn gesehen hatten?

Aber, wird man fragen, wie kam denn Irenaeus dazu, den Papias als Hörer des Johannes zu bezeichnen, wenn er sich nicht selber so in seinem Werke irgendwo genannt hatte? Es könnte vermutet werden, dass Irenaeus gar nicht an den Apostel Johannes gedacht habe, sondern an den andern Johannes, den Papias in der Vorrede nennt und den er gleichfalls als einen Herrenschüler bezeichnet. Aber Irenaeus ignoriert diesen zweiten Johannes vollständig, er spricht immer nur von einem Johannes und lässt keinen Zweifel, dass er damit den Apostel meint. Denn wenn er auch von ihm immer nur als dem Schüler des Herrn spricht, den die Presbyter gesehen haben, so stellt er ihm an einer Stelle die übrigen Apostel gegenüber (II; 22, 5), ein deutliches Zeichen, dass der Gedanke an einen andern Johannes bei ihm ausgeschlossen ist.

Aber Irenaeus ist nicht der einzige, der Papias' Verkehr mit Johannes bezeugt, vielmehr citiert kaum ein kirchlicher Schriftsteller seinen Namen ohne einen Zusatz, der sein enges Verhältnis zu dem Apostel ausdrückt. Dies verdient weiter keine Beachtung ausser in den Fällen, wo es von solchen geschieht, die Papias selbst gelesen hatten.

Aber auch ihr Zeugnis hat keinen weiteren Wert, wenn sie sich von Irenaeus direct, wie Anastasius Sinaita, oder indirect - durch Vermittlung des Eusebius - wie Philippus Sidetes, beeinflusst zeigen. Anders steht es mit dem Zeugnis des Apolinarius (s. Preuschen, Antileg. S. 6r), wenn dieser mit dem Bischof von Hierapolis identisch sein sollte. Allein da sich dies nicht ausmachen lässt (s. Harnack, Patres apost. I, 2, 93), 
so wird man Irenacus dafür verantwortlich machen dürfen und müssen, dass die Folgezeit den Papias für einen Schüler des Johannes gehalten hat. Geirrt hat er sich darin ohne Zweifel, wenn sich auch die Entstehung seines Irrtums nicht nachweisen lässt.

Wie mächtig die Autorität des Irenacus gewirkt hat, mag man daraus ermessen, dass derselbe Eusebius, der in der h. e. seine Behauptung bezweifelt, sic in der Chronik als Thatsache aufstellt (,nach Johannes wurden Papias von Hierapolis und Polykarp, Bischof von Smyrna, seine Hörer, bekannt", s. Preuschen, Antil. S. 54).

Wir haben noch den Schluss des Prooemiums zu prüfen, in dem Papias von dem, was er an Aussprüchen der Apostel erkundet hat, das scheidet, was Aristion und der Presbyter Johannes sagen. Mit Fug und Recht bemerht Eusebius, dass Papias diesen Johannes dadurch, dass er ihn ausserhalb der Reihe der Apostel setzè und ihn als Presbyter bezeichnet, von dem Evangelisten unterscheiden wolle. Auffällig ist nur, dass Aristion und der Presbyter Johannes, während sie von den Herrenschülern unterschieden werden, doch zugleich selbst als Herrenschüler bezeichnet sind. Der Gewaltakt, durch den man diesem Umstand hat begegnen wollen, indem man den ersten Johannes aus der Reihe der Apostel strich ${ }^{x}$, damit der Presbyter als dieser erschiene, lässt nicht einmal eine Discussion zu. Denn wie wäre es, um nur dies hervorzuheben, denkbar, dass der eine unbekannte Aristion auf eine Stufe mit den Uraposteln gestellt wäre? Eher könnte man daran denken, dass durch ein Versehen des Eusebius oder seiner Abschreiber Aristion und der Presbyter Johannes als Herrenschüler bezeichnet seien. Wenigstens scheint Papias nach dem Beispiel, das Eusebius giebt (III, 39, 15), den zweiten Johannes für gewöhnlich einfach als Presbyter und nicht auch,als Herrenschüler citiert $z \mathrm{u}$ haben. Wenn Eusebius sagt, Papias behaupte (甲ncí), Aristion und den Presbyter Johannes selbst gehört zu haben, so meint er dies doch nicht so, als wenn Papias dies irgendwo ausdrücklich erkläre, sondern er schliesst es nur daraus, dass er diese beiden oft namentlich citierte ${ }^{2}$. Hieraus folgt nur, dass die andern Presbyter bei ihm ebenso unbenannt geblieben waren, wie sie es bei Irenaeus sind. Halten wir uns an Papias' eigene Worte, so hatte er auch das, was er von ihnen wusste, durch die Vermittlung derselben Leute, die ihm aus dem Munde

I So Haussleiter, Theol. Litteraturbl. 1896, Sp. 465 ff.

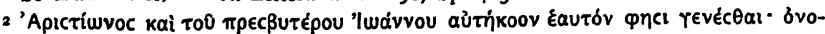

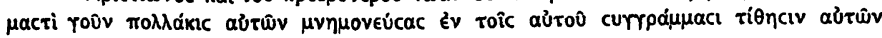

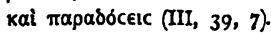


der Presbyter die Aussprüche der Apostel berichteten, nur mit dem Unterschiede, dass er annahm, diese beiden seien noch am Leben gewesen, als er seine Erkundigungen einzog ${ }^{x}$. Er verdankte, wie Eusebius sagt, dem Aristion Erläuterungen der Reden des Herrn, dem Johannes Überlieferungen, von denen uns Eusebius die bekannten Proben über die Entsttehung des Marcus- und Matthäusevangeliums bietet. Stammen diese Bezeichnungen aus dem Werke des Papias, was man freilich nicht mit Sicherheit behaupten kann, so kann man kaum umhin, trotz seiner eigenen Versicherung an Büchertitel zu denken² (stimmt doch die erste fast wörtlich mit dem Titel seines eigenen Werkes), in welchem Falle man dann Aristion als seinen litterarischen Vorgänger $z \mathbf{u}$ betrachten hätte. Wie dem aber auch sei und ob nun Papias den Aristion und den Presbyter Johannes für Herrenschüler gehalten haben mag oder nicht, so zeigt jedenfalls die Unterscheidung, die er macht, dass er ihre Überlieferungen anders bewertet.

Hält man dies fest, so ist es schwer zu begreifen, wie man zu der Meinung hat kommen können, die auch Harnack in seiner Chronologie zu der seinen gemacht und neu zu stützen und begründen gesucht hat, dass Irenaeus eine Verwechselung des Apostels und des Presbyters Johannes begangen habe. „Unstreitig“, so etwa sagt Harnack S. 668, „meint Irenaeus an den fünf Stellen, an denen er nach Papias über Johannes referiert, den Zebedaïden, aber er bezeichnet ihn doch nur als Herrenschüler. Die Stellen können also genau so, wie sie lauten, von Papias übernommen sein, und Papias kann doch nicht den Zebedaïden, sondern den Presbyter gemeint haben. Entscheiden lässt sich das nicht."

Ich glaube, das lässt sich doch entscheiden. Auch Eusebius, der den Papias gelesen und mit Kritik gelesen hat und der den Irenaeus beschuldigt, sich durch ihn zu falschen Meinungen haben verführen zu lassen, hat unter dem Herrenschüler Johannes, auf den Papias die Schilderungen von dem tausendjährigen Reiche zurückführte (Iren. V, 33, 3), den Apostel Johannes verstanden. Denn er sagt, dass Papias' Vorstellungen von dem tausendjährigen Reiche auf Missverständnis der a postolischen Erzählungen beruhen (h. e. III, 39, I2 u. I3). Wir wissen aber auch aus Eusebius, dass Papias, wenn schon er den Pres-

I Ich verstehe, wie Harnack, Chronol. S. 660 Anm. 2, die Worte des Prooemiums

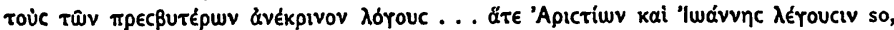

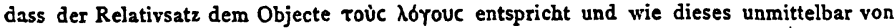

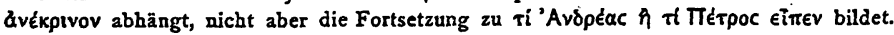

2 Dieser Meinung ist auch Harnack, Chronol. S. 698, Anm. 1. 
byter Joliannes in seinem Prooemium als Herrenschüler bezeichnete, er iln doch in seinem Werke als Presbyter citierte. Es ist zwar nicht mit mathematischer Gewissheit auszumachen, ob er es jedesmal that, aber da $\mathrm{er}$ in dem Prooemium den Presbyter Johannes als Quelle neben den unbenannten Presbytern aufführt und da er die Überlieferungen des Presbyters nach dem Zcugnis des Euscbius unter dessen Namen citiert, so kann er doch nicht da, wo er die unbenannten Presbyter von Johannes erzählen lässt, sie über den Presbyter, sondern nur über den Apostel Johannes berichten lassen.

Eusebius gründet auf die Unterscheidung der beiden Johannes bei Papias die Vermutung, dass die Apokalypse von einem andern Verfasser als das Evangelium sei. „Wenn man nicht annehmen will, dass der erste der beiden Johannes die unter dem Namen des Johannes umlaufende Apokalypse gesehen hat", sagt er, "so ist es vermutlich der zweite". (h. e. III, 39, 6). Schon die Form, in der diese Vermutung auftritt, zeigt, dass sie keinerlei Anhalt in dem Werke des Papias gehabt haben kann. Sie beruht lediglich auf der Gleichheit der Namen und ist, wie sich später herausstellt, von Eusebius dem Dionysius von Alexandria entlehnt. Dieser stützte sich seinerseits darauf, dass es in Ephesus zwei Grabmäler gäbe, die beide die des Johannes genannt würden (h. e. VII, 25, 16). Er sagt nicht, dass sie als das des Apostels und des Presbyters unterschieden worden wären. Er weist auch nicht selbst das eine diesem, das andere jenem zu. Daraus geht hervor, dass er von dem Presbyter des Papias nichts weiss. Erst Eusebius hat die von Dionysius angeführte Thatsache mit Papias combiniert. Mit Recht nennt Harnack die Angabe des Dionysius ein ganz schwaches Argument (S. 662, Anm. I). Nur verstehe ich nicht, wie er dann auf Grund eben dieses Arguments das Schweigen des Polykrates von Ephesus über den Presbyter Johannes verwunderlich findet (S. 669). Wenn man im Anfang des 3. Jahrhunderts wirklich zwei Gräber des Johannes gezeigt hat, so bedeutet das doch nicht, dass wirklich zwei verschiedene Johannes in Ephesus begraben waren, was lediglich Vermutung des Dionysius ist, noch weniger, dass der eine der Apostel, der andere der Presbyter war, was erst Eusebius

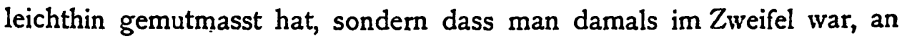
welcher Stelle der Apostel begraben lag, indem die einen diese, die andern eine andere dafür hielten. Jedenfalls aber darf man doch nicht eine Vermutung des 3. Jahrhunderts gegen eine Behauptung des 2. ausspielen. Wenn also Polykrates von Ephesus unter den grossen Sternen der kleinasiatischen Kirche nur den einen Apostel und Evangelisten 
Johannes nennt, der in Ephesus begraben sei, so hat er eben nur diesen einen Johannes als grosses Licht gekannt und von dem Presbyter entweder überhaupt nichts gewusst oder doch nichts, was ihn einer Erwähnung in solchem Zusammenhange hätte würdig erscheinen lassen. Was aber nötigt oder berechtigt uns, die wir von diesem Presbyter nichts w'eiter kennen als die kümmerlichen Angaben, die uns Eusebius über ihn aus Papias erhalten hat, ihn, wie Harnack thut, für eine Säule der Kirche zu halten? Ist seine Charakteristik des Evangelisten Marcus etwa derart, dass wir ihn darum für eine bedeutende Persönlichkeit erklären müssten?

Wir haben gesehen, dass an den Stellen, an denen Papias die Presbyter von dem Herrenschüler Johannes berichten lässt, er damit den Apostel meint. Darum bliebe nun freilich, solange nicht bewiesen ist, dass er hier zugleich von dem Evangelisten spricht, immerhin noch Raum für die Annahme, dass er den Presbyter Johannes für den Verfasser des vierten Evangeliums gehalten habe. Aber gesetzt, er habe das gethan, sollte davon weder Irenaeus noch Eusebius etwas gemerkt haben? Und wenn Irenaeus es vielleicht absichtlich ignoriert haben könnte, würde Eusebius nicht mit Begierde die Gelegenheit ergriffen haben, den nach seinem Utteil beschränkten Mann noch weiter zu discreditieren? Denn darüber kann doch wohl kein $Z_{\text {weifel sein, dass }}$ Eusebius eine solche Meinung für eine eben so schlimme Ketzerei wie die chiliastischen Träumereien des Papias gehalten haben würde.

Wenn wir es also einstweilen noch dahin gestellt sein lassen, ob Papias schon den Apostel Johannes als den Evangelisten gekannt hat, so dürfen und müssen wir schon jetzt auf, das bestimmteste erklären, dass er so wenig wie Irenaeus und Polykrates noch irgend sonst einer aus dem 2. Jahrh. den Presbyter Johannes dafür gehalten hat. Ebenso ist daran festzuhalten, dass auf Grund von Papias eine Übertragung von Zügen des Presbyters auf den Apostel nicht stattgefunden haben kann. Es giebt also kein äusseres Zeugnis, dass jemals eine Verwechselung des Presbyters und Apostels Johannes stattgefunden habe, sondern alles, was dafür gehalten wurde, löst sich als Schein auf, der aus der gänzlich vagen und gar nicht einmal ernst gemeinten Vermutung des Eusebius entstanden ist.

Was aber die inneren Zeugnisse für die angenommene Verwechselung betrifft, die Harnack S. 675 ff. entwickelt, so lassen sich diese auf folgende drei Sätze reducieren:

I. Der Verfasser des 2. und 3. Johannesbriefes nennt sich $\delta \pi \rho \in c \beta u ́-$

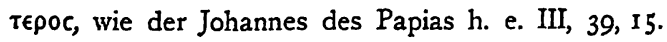


2. Der 2. und 3. Johannesbrief lassen sich nicht von dem ersten, dieser nicht von dem Evangelium trennen.

3. Folglich ist der Presbyter Johannes des Papias der Verfasser des Evangeliums.

Von diesen Sätzen liegt, wie man sieht, der zweite ganz und gar auf dem Boden der inneren Kritik, und diesen zu betreten verbieten die Grenzen dieses Aufsatzes. Aber klar ist, dass sich schon vom rein formalen Standpunkt aus schwerwiegende Bedenken erheben. Denn, wie schon Dionysius von Alexandria bemerkt (h. c. VII, 25, II), so steht in dem 2. und 3. sogenannten Briefe des Johannes nicht Johannes namentlich voran, sondern es ist ohne Namen "der Presbyter" geschrieben. Daraufhin aber die Identificierung dieses Presbyters mit dem Presbyter Johannes vorzunehmen, ist gewagt, denn es gab der Presbyter gar manche und dass der Presbyter Johannes als der Presbyter kat' Ėoxìv berühmt und kenntlich war, ist vielmehr Gegenstand ais Voraussetzung der Beweisführung. Gesetzt also auch, dass Satz 2 unanfechtbar wäre, so würde man doch nach den Gesetzen der Logik nur zu dem Schlusse kommen, dass das Evangelium von einem Presbyter, nicht aber dass es von dem Presbyter Johannes verfasst sei.

Können nun die Ansprüche des Presbyters Johannes auf die Autorschaft des vierten Evangeliums als beseitigt gelten und steht es fest, dass die Überlieferungen der Presbyter über den Herrenschüler Johannes bei Irenaeus sich auf den Apostel beziehen, so wäre von dieser Seite der Weg zu der Frage offen, ob in jenen Aussagen irgend welche Anzeichen von dem Vorhanden- oder Nichtvorhandensein einer Tradition über die Entstehung des Evangeliums zu entdecken sind.

Von dieser Frage verschieden und doch aufs engste mit ihr verknüpft ist die Frage, ob Papias und seine Gewährsleute das vierte Evangelium gekannt haben. Es ist die Vorfrage, die wir zuerst ins "Auge zu fassen haben.

Die Meinungen darüber stehen sich mehr oder minder schroff gegenüber. Ich selber habe es früher zwar nicht für ausgeschlossen gehalten, dass Papias das vierte Evangelium gekannt, aber doch für überwiegend wahrscheinlich, dass er es nicht gekannt habe. Seitdem habe ich mich, besonders durch die von Lightfoot schon 1875 vorgebrachten Gründe überzeugen lassen, dass die Sache gerade umgekehrt liegt ${ }^{x}$.

Lightfoot hat das grosse Verdienst, eine richtige Vorstellung von

I Contemporary Review, Bd. 26, p. 377 ff. und $\$ 28$ ff. Papias of Hierapolis. 
dem Wesen und der Tendenz des papianischen Werkes gegeben zu haben, die von so entgegengesetzten Forschem wie Zahn (Kanonsgesch. S. 849 ff.) und Harnack (Chronologie, S. 663) gleichmässig angenommen ist. Wenn Papias sagt, dass er bestrebt gewesen sei, nicht sowohl aus den Büchern als aus dem lebendigen Worte Nutzen zu ziehen, so folgt daraus weder, dass er unsere Evangelien nicht gekannt, noch auch, dass er ihnen die mündliche Überlieferung vorgezogen habe. Er veranstaltete keine neue Sammlung von Herrenaussprüchen, sondern er gab Erläuterungen davon, wobei er allerdings auch, wenn er konnte, nicht aufgezeichnete Aussprüche des Herrn mitteilte, wie ein uns erhaltenes Beispiel zeigt. Aber gerade dieses Beispiel zeigt auch, wie Lightfoot trefflich auseinandergesetzt hat (S. 389 ), dass solche angeblich mündlich überlieferten Aussprüche nur zur Erläuterung von schriftlichen dienten. Die Herrenaussprüche werden also als gegeben vorausgesetzt, Papias aber sucht sie so viel wie möglich aus der mündlichen Tradition zu erläutern, wobei dann freilich noch manches zur Erörterung kam, was diesem Zwecke keineswegs unmittelbar diente. Diese seine Erläuterungen setzt er denen der Schwätzer, die fremde Gebote überliefern, entgegen. Damit sind offenbar Haeretiker gemeint, die sich nicht von der als echt geltenden Tradition leiten lassen. Treffend sagt Lightfoot (S. 390/r), dass Irenaeus in den Gnostikern gewissermassen das Widerspiel des Papias zeichne, wenn er im Anfang seines grossen Werkes von ihnen als von schlechten

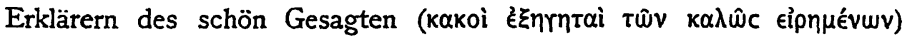
spreche.

Wenn nun Papias ohne Zweifeł Evangelien kannte und benutzte, so fragt sich doch, welche. Mit Sicherheit kann man als solches nur das Marcusevangelium nennen, denn dafür, dass das Evangelium, welches er unter diesem Namen anführt, ein anderes als das uns erhaltene sei, lässt sich kein Beweis erbringen. Mit hoher Wahrscheinlichkeit wird man es auch von dem Matthäusevangelium annehmen können, obwohl er anscheinend mehrere griechische Recensionen davon kennt. Anders steht es mit Lucas und Johannes. $\mathrm{Ob}$ er sie namentlich erwähnt hat oder nicht, ist uns nicht überliefert.

Von Lucas sehen wir ab und fragen nach Johannes.

Ein indirectes Zeugnis, dass Papias das Evangelium gekannt hat, liegt jedenfalls in seiner von Eusebius bezeugten Bekanntschaft mit dem 1. Johannesbrief (h. e. III, 39, 17). Auch an directen Anzeichen scheint es nicht zu fehlen. $\mathrm{Da}$ ișt zunächst die Reihenfolge, in der die Apostel in dem Prooemium aufgeführt waren: Andreas, Petrus, Philippus, Thomas, 
Jacobus, Johannes, Matthüus. Dass Petrus an zweiter Stelle zwischen Andreas und Philippus stelit, erscheint bei Bckanntschaft mit dem Johannesevangelium nicht unnatürlich. Auch die Zusammenstellung, Johannes und Matthäus, wird erklärlich, wenn Papias Johannes wie Matthäus für den Verfasser cines Evangeliums hielt. Ferner hat die Gleichsetzung

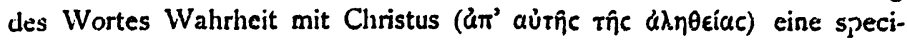
fisch johanneische Färbung ${ }^{3}$.

Aber bedeutsamer ist doch noch das Zusammentreffen des Papias mit dem Johannesevangelium an zwei andern Stellen. Nach Irenaeus V, 36, 2 citierten die Presbyter ein Herrenwort, das sich mit dem Ausspruch: "in dem Hause meines Vaters sind viele Wohnungen" Joh 14, 2 deckt.

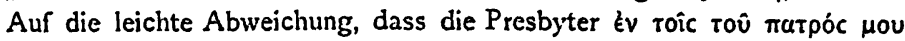

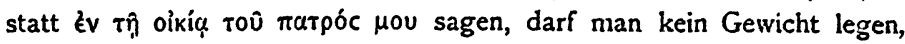
denn sie wurde durch den Zusammenhang, in dem sie das Wort gebrauchten, gefordert, da sie im Jenseits die Himmel, das Paradies und das neue Jerusalem unterschieden und bei ihrer grobsinnlichen Auffassung das alles zusammen nicht gut als das Haus bezeichnen konnten. Nun wäre es ja freilich möglich, dass dies Wort, wie mehrfach angenommen ist, nicht erst von dem Verfasser des vierten Evangeliums geprägt ist und also die Presbyter es auch von anderer Seite empfangen haben können. Aber gegenüber der Thatsächlichkeit der Übereinstimmung der Presbyter mit dem vierten Evangelium hat die vage Möglichkeit ihrer Abhängigkeit von einer anderen Quelle nicht eben allzuviel Ửberzeugungskraft in sich.

Eine umständlichere Erörterung verlangt die zweite Stelle, an der die Presbyter sich mit dem vierten Evangelium berühren. Irenaeus behauptet, die Presbyter bezeugten, ebenso wie das Evangelium $(8,57)$, Jesus habe in einem höheren Alter gelehrt, und zwar habe ihnen eben dies Johannes überliefert, mit dem sie bis zu den Zeiten Trajans" in Asien zusammen gewesen wären (II, 22, 5). Er stellt also das Zeugnis der Presbyter neben das Evangelium, als wenn es zwei verschiedene Zeugnisse wären, obwohl es doch für ihn das Zeugnis desselben Mannes war. Hätte er sagen wollen, die Presbyter hätten bezeugt, Johannes hätte ihnen mündlich das bestätigt, was er in seinem Evangelium schriftlich niedergelegt hatte, so hätte er das mit einem Wörtchen ausdrücken können. Man kann also nicht umhin anzunehmen, dass in seiner Quelle des Evangeliums entweder überhaupt nicht oder doch nicht als des

× Nach Lightfoot S. 839 . 
Evangeliums des Johannes in diesem Zusammenhange Erwähnung gethan war, wie denn ja auch das Zeugnis der Presbyter erst dann überhaupt Gewicht hat, wenn es von dem Evangelium unabhängig erscheint. Es ist damit freilich nicht gesagt, dass die angebliche Bekundung eines höheren Alters des Herrn durch die Presbyter thatsächlich nicht doch lediglich auf dem vierten Evangelium beruhe, eine Annahme, die allerdings die Voraussetzung in sich schliesst, oder vielmehr auf ihr beruht, dass diese Presbyter den Apostel gar nicht persönlich gekannt hatten. Die Berechtigung eben dieser Annahme haben wir zu untersuchen.

Für zweifellos unabhängig von dem Evangelium müsste das Zeugnis der Presbyter gelten, wenn es etwas ganz anderes besagte als dieses selbst. Ich habe das in den Monarchianischen Prologen S. 107 f. zu beweisen versucht und in demselben Sinne hat sich dann Harnack in der Chronologie S. 335 Anm. ausgesprochen. Aber ich glaube, diese Meinung hält nicht Stand. Allerdings drückt Irenaeus sich so gewunden aus und presst, um Jesus einen Anteil an der letzten Stufe des Alters zuschreiben zu können, was wohl die Presbyter thaten, aber nicht das Evangelium, den Ausdruck des Evangeliums: du bist noch nicht fünfzig Jahr, derart, dass man unwillkürlich den Eindruck gewinnt, als suche er zwei verschiedene Angaben künstlich mit einander auszugleichen. Aber man muss sehr behutsam sein, wenn man Irenaeus auf dem schlüpfrigen Boden folgen will, auf dem er sich bewegt. Es erscheint von vornherein kaum glaublich, wie die Presbyter den Mut gefunden haben sollten, eine von aller Überlieferung so verlassene Behauptung aufzustellen, wie die, dass Jesus ein Greis im eigentlichen Sinne geworden sei. Aber thatsächlich schiebt Irenaeus ihnen eine solche Behauptung auch gar nicht zu. Er sagt nur, dass sie Jesus ein höheres Alter, eine aetas senior beigelegt hätten, das ist aber noch nicht die eigentliche senectus, ein Ausdruck, den Irenaeus in diesem ganzen Abschnitt mit gutem Grunde meidet. Er unterscheidet drei Stufen des menschlichen Lebens: Kindheit, Jugend und höheres Alter. Bei dieser Einteilung muss ein den Fünfzigen nahe stehender Mann natürlich zu den Älteren gerechnet werden. Irenaeus meint, der Begriff der menschlichen Vollkommenheit verlange, dass Jesus auch die höchste Stufe des Lebens erreicht haben, und dass der, durch den die Alten wie die Jungen gerettet würden, auch ein Greis unter Greisen gewesen sein müsse. Er vergisst dabei, dass dies Argument durchschlagend nur wäre, wenn Jesus den letzten Lebensabschnitt, wie die übrigen, ganz durchmessen, nicht nur seine Schwelle eben überschritten gehabt hätte. Aber er ist zufrieden, Jesus überhaupt 
unter die Älteren einreihen zu können, und er behauptet auch von den Presbytern nichts anderes. Wir sind daher nicht zu der Annahme berechtigt, dass sie den Ausdruck senior in einem anderen als diesem relativen Sinne gebraucht hatten. Es lässt sich dies aber auch positiv beweisen, denn Irenacus ist hicr von den Presbytern oder vielmehr von I'apias weit mehr abhängig als es auf den ersten Blick scheint.

Die Voraussetzung, dass Jesus annähernd fünfzig Jahr alt geworden sei, konnte offenbar nicht mehr gemacht werden, wenn das erste und dritte Evangelium kanonische Geltung hatten. Um so übler war man daran, als alle vier diese Geltung erlangt hatten und nun auf dem Wege der Interpretation der Widerspruch zwischen dem vierten und ersten und dritten Evangelium aufzuheben war. Dies geht natürlich nur so, dass man entweder dem vierten oder dem ersten und dritten Gewalt anthut. Die Neueren pflegen den ersten Weg zu beschreiten, Irenaeus entschied sich für den zweiten. Er sagt ganz richtig, das Johannesevangelium drücke sich so aus, dass man annehmen müsse, Jesus habe das vierzigste Jahr längst überschritten, das fünfzigste aber noch nicht ganz erreicht (II, 22, 6), er nimmt mit Lucas an, dass Jesus mit annähernd 30 Jahren zur Taufe gekommen sei $(22,5)$ dann aber behauptet er, um beide Evangelien zu vereinigen, zwischen der Taufe und dem Tode Jesu liege ein Zeitraum von annähernd 20 Jahren $(22,6 \text { g. E. })^{x}$. Sollte Irenaeus auf diesen ungeheuerlichen Gedanken ganz selbständig gekommen sein? Jedenfalls gerät er auf diesem Ausweg der Verzweiflung mit sich selbst in einen flagranten Widerspruch. Gegen die Ansicht, dass Jesus nur ein Jahr gelehrt habe, hatte Irenaeus sich darauf berufen, dass Jesus nach dem Johannesevangelium dreimal nach seiner Taufe zum Passahfeste nach Jerusalem gereist sei $(22,3)$. Diese Reisen denkt er sich in den drei auf die Taufe folgenden Jahren ausgeführt, denn er sagt ausdrücklich, Jesus habe die jüdische Sitte befolgt, jedes Jahr um diese Zeit nach Jerusalem zu ziehen. Das hat er wenige Abschnitte später, wo er mit der Thatsache rechnet, dass Jesus bei seiner Taufe doch erst dreissig Jahr alt gewesen sei, völlig wieder vergessen.

Er verwickelt sich aber noch in einen andern Widerspruch. Neben der Forderung, dass Jesus, um das Gesetz der Menschlichkeit ganz zu erfüllen, auch den letzten Lebensabschnitt habe erreichen müssen, hat Irenaeus noch ein zweites Argument, mit dem er die Voraussetzung des

I Non ergo anno uno praedicavit nec duodecimo mense anni passus est. Tempus enim a trigesimo anno usque ad quinquagesimum nunquam erit unus annus.

6. 8. xgor. 
vierten Evangeliums als richtig zu erweisen sucht: um als Lehrer aufzutreten, musste Jesus auch das Alter des Lehrers haben; wie hätte er ohne dies zu haben, lehren sollen? $(22,5)$. Sollte dieses Argument bestehen, so musste Irenaeus entweder die Zeitangabe des Lucas, dass Jesus bei der Taufe 30 Jahre alt gewesen sei, widerlegen, oder er musste im Widerspruch mit allen vier Evangelien behaupten, dass Jesus erst lange Zeit nach der Taufe zu lehren angefangen habe. Dagegen nimmt er das Datum des Lucas an und lenkt die Aufmerksamkeit von der Kluft, die seine Argumentation zerreisst, durch einen übel angebrachten Angriff gegen seine Gegner ab. „Während er mit 30 Jahren zur Taufe kam, ging er hernach, als er das vollkommene Alter des Lehrers hatte, nach Jerusalem, so dass er mit Recht von allen Lehrer genannt wurde: denn er schien nichts anderes als er war, wie die behaupten, die ein Scheinwesen einfuhren; sondern was er war, das schien er auch. Als er daher als Lehrer auftrat, hatte er auch das Alter eines Lehrers" $(22,4)$.

Man sieht, dass Irenaeus mit Argumenten arbeitet, die mit der Anerkennung des ersten und dritten, ganz besonders aber des dritten Evangeliums unverträglich sind. Es ist der verzweifelte Versuch; verschiedene Anschauungen zu vereinigen. Die Argumente, deren Irenaeus sich bedient, sind vollkommen begreiflich auf einem Standpunkt, der an das Lucasevangelium noch nicht gebunden ist, sei es, dass man es noch nicht kennt, oder, was ziemlich auf dasselbe hinauskommt, es anzuerkennen sich noch nicht verpflichtet fiuhlt. Es scheint mir daher sicher, dass Irenaeus auch seine Argumente von Papias entlehnt hat.

Es kommt nun aber nicht soivohl darauf an, welches Alter Papias oder seine Presbyter Jesus beigelegt, als in welche Zeit sie seinen Tod gesetzt haben, denn dass sie auch darin von der allgemeinen Annahme abgewichen seien, ist damit nicht gesagt, dass sie sein Alter höher bemassen als es bei Lucas geschieht. Der vierte Evangelist, wenn anders er sich überhaupt Gedanken darüber gemacht hat, hat schwerlich Jesu Tod I8 Jahre später als. Lucas ansetzen wollen. Irenaeus freilich thut es indirect, wenn er sich auch hütet es auszusprechen. Aber wenn er von Papias abhängt, Papias aber auf Lc 3, 23 keine Rücksicht nahm, so braucht von diesem nicht dasselbe gegolten zu haben.

Ein Anhalt für die Zeitbestimmung war in der Überlieferung gegeben, dass Jesus unter Pontius Pilatus gestorben sei. Aber in welchem Jahre seiner Verwaltung dies geschehen, oder auch in welche Zeit diese Verwaltung überhaupt gefallen sei, darüber finden wir weder bei Marcus noch bei Matthäus noch bei Johannes irgend eine Angabe. Lucas ist 
für uns der erste, der das Jahr genau zu bestimmen gesucht hat. Er thut es mit einem solchen Aufwand von Daten, dass man sieht, wie viel ihm daran gelegen ist, jeden Zweifel auszuschliessen. Er mochte seine Grunde dazu haben. Je weiter man sich von den ersten Zeiten cntfcrnte, um so unsicherer mussten dic Vorstellungen von der Folge der Zeiten werden. Dies aber konnte höchst bedenkliche Consequenzen haben, weil man, wic Papias und auch noch Irenaeus zeigt, sich von der mündlichen Uberlieferung noch nicht lossagen mochte, als ihr Wert doch schon sehr fragwürdig erscheinen musste. Die Angabe, dass Johannes bis Trajan gelebt habe, bleibt, auch unter der Voraussetzung, dass Jesus im Jahre 29 gekreuzigt sei, in den Grenzen des Möglichen. Bedenklicher ist es, wenn Papias den Aristion und den Presbyter Johannes, die er doch den Presbytern, wclche mit den Aposteln verkehrt haben sollten, gleichstellt, ebenfalls Jünger des Herrn nennt. Kann man aber annehmen, dass dieser Papias richtige chronologische Vorstellungen gehabt habe, wenn er erzählte, dass von Christus auferweckte Tote bis Hadrian gelebt hätten? ${ }^{2}$

Führen daher schon allgemeine Erwägungen zu der Vermutung, dass die chronologischen Vorstellungen von dem Tode Jesu in der ersten Hälfte des zweiten Jahrhunderts sich bedenklich zu verschieben anfingen, so darf man nicht an der merkwürdigen Notiz eines Anonymus aus dem Jahre 810 vorübergehen, die folgendermassen lautet ${ }^{2}$ : In commentariis Victorini inter plurima haec etiam scripta reperimus. Invenimus in membranis Alexandri episcopi qui fuit in Hierusalem, quod transscripsit manu sua de exemplaribus apostolorum. Ita VIII. Kal. Ianu. natus est dominus noster Iesus Christus Sulpitio et Camerino Coss. et baptizatus est VIII. Id. Ian. Valeriano et Asiatico Coss., passus est X. Kal. Apr. Nerone III. et Valerio Messalla Coss., resurrexit VIII. Kal. April. Coss. supra scriptis, ascendit in coelos V. Non. Mai. post dies XL. Ćoss. supra scriptis. Ioannes baptista nascitur VIII. Kal. Iul. et circumciditur Kal. Iul., ad Mariam vero locutus est angelus VIII. Kal. Apr. sexto iam conceptionis mense Elisabeth [in utero] habere dicens.

Aus Namen und Beinamen sind in den beiden ersten Fällen zwei Consuln gemacht und im zweiten ist Valerianus aus Valerius geworden, sonst aber sind sie richtig und ergeben die Jahre 9, 46 und 58. Ohne Zweifel erregt die Angabe der Monatstage in dieser Notiz den Verdacht,

I Preuschen, Antil. S. $5^{8 .}$

2 Vgl. Harnack, Altchristl. Litteraturgesch. S. 506. v. Dobschütz, D. Kerygma Petri (Texte u. Unters. XI, I) S. 137. 
dass sie nicht so alt ist wie sie $\mathrm{zu}$ sein behauptet. Aber es ist die Frage, ob dàs nicht spätere Zusätze sind. Die Angabe über die Geburt des Täufers und die Empfängnis der Maria scheint sich als solchen dadurch $z$ verraten, dass hier das Consulat nicht beigefügt ist. Man darf nicht sagen, dass schon dem ursprünglichen Verfasser der Notiz das überfüssig erscheinen mochte, denn es ist das Consulat auch zu der Auferstehung und Himmelfahrt beigesetzt, wo es sich doch auch von selbst ergab. Wie dem aber auch sei, so lässt sich jedenfalls nicht begreifen, wie und warum man in späterer Zeit auf die angegebenen Consulate hätte verfallen sollen. Ich kann mir nicht vorstellen, wie jemand, nachdem das Lucasevangelium kanonische Geltung gewonnen hatte, es hätte wagen können zu behaupten, Jesus sei unter Nero gekreuzigt worden. Auch Irenaeus hütet sich wohl, es gerade herauszusagen, dass die Kreuzigung Jesu erst unter Claudius stattgefunden habe, obwohl es aus seinen Worten folgt, und seine gewundene Redeweise ist Beweis genug, wie vorsichtig er sein musste, um nicht den Widerspruch geradezu herauszufordern. Man muss daher die Angabe der Consulate als den Kern einer alten Nachricht anerkennen, die wirklich auf dem angegebenen Wege dem Anonymus zugekommen ist. Ganz unklar ist dabei freilich, was denn des Bischofs Alexander ${ }^{x}$ Quelle war. Aber soviel geht doch aus dem Ausdruck hervor, dass er diese Überlieferung auf die Apostel zurückführte. Das alles weist geradezu auf Papias hin. Bei den Commentaren des Victorin kann man nur an Victorinus von Pettau denken, von dessen Commentar zur Apokalypse Haussleiter in dem Theol. Litteraturbl. $1895 \mathrm{Sp} .193 \mathrm{ff}$. den chiliastischen Schluss in seiner ursprünglichen, von Hieronymus noch unangetasteten Gestalt, veröffentlicht hat. Die Anklänge an Papias sind darin so deutlich wie möglich. Sollte Alexander einen Auszug aus Papias verfertigt und diesen Victorinus benutzt haben?

Es lässt sich aber auf einem andern Wege fast zur Gewissheit erheben, dass die aus Victorinus geschöpften chronologischen Daten auf Papias zurückgehen. Der Leser möge die Geduld nicht verlieren, wenn ich zu diesem $Z$ weck noch einmal zu Irenaeus zurückkehre. Wir haben oben (S. 217) gesehen, wie Irenaeus sich durch seine Forderung, Jesus müsse als Lehrer auch das Alter eines Lehrers gehabt haben, in einen unauflöslichen Widerspruch verwickelt, da er die Erzählung des Lucas anerkennt, dass Jesus mit dreissig Jahren von Johannes getauft sei, und

I Er gehört der ersten Hälfte des 3. Jahrh.s an, s. Harnack, Altchr. Litteratgesch. S. 505. Dass er der Begründer der Bibliothek 2u Jerusalem war, sagt Eusebius h. e. VI, 20. 
die einstimmige Úberlieferung der Evangelien, dass er alsbald nach seiner Taufe $z u$ lehren angefangen habe, nicht zu bestreiten wagt. Nachdem er seinen Gegnern vorgerückt hat, dass, wenn sie annehmen, Jesus habe nur ein Jahr nach seincr Taufe gepredigt, er noch als Jüngling, ehe cr ein vorgerücktes Alter erreicht habe, gestorben sei, fährt er so fort: Quia autem triginta annorum aetas prima indolis est iuvenis et extenditur usque ad quadragesimum annum, omnis quilibet confitebitur; a quadragesimo autem [et quinquagesimo] anno declinat iam in aetatem seniorem, quam habens Dominus noster docebat (II, 22, 5).

Der Ausdruck indoles wird von Irenaeus mit Rücksicht darauf gewählt sein, dass nach seiner Meinung das Lehramt der jugendlichen Anlage und Befähigung (iuvenis ist offenbar adjektivisch zu verstehen) nicht entspricht. Er hatte ja, wie wir sahen, erklärt, dass ein Dreissigjähriger zum Lehrer noch nicht geeignet sei, und er hatte seine Gegner getadelt, dass sie Jesus das ehrenvollere, vorgerücktẻ Alter nähmen, in dem er als Lehrer alle überragte. " Hier handelt es sich darum, zu beweisen, welches dieses vorgerückte Alter ist. Offenbar will Irenaeus sagen: mit dreissig Jahren ist der erste Abschnitt der Jugend zurückgelegt, aber sie hat damit noch nicht ihr Ende erreicht, sondern dehnt sich bis zum vierzigsten Jahre aus. Nun beginnt die Neige zum Alter. Was aber soll a quadragesimo autem et quinquagesimo anno heissen? Die Neige zum Alter beginnt entweder mit dem vierzigsten oder dem funfzigsten Jahre, unmöglich können beide gleich gesetzt werden. Die Lesart et quinquagesimo ist daher sicher falsch, aber auch mit der Lesart aut quinquagesimo, die sich daneben findet, ist nichts anzufangen. Wenn Irenaeus die Jugend bis zum 40. Jahre rechnet, wie er es thut, so muss er auch in eben dieses Jahr den Wendepunkt zum Alter setzen. Würde er dies erst beim 50. Jahre beginnen lassen, so würde er seine Beweisführung selbst aufheben, da ja nach seiner Annahme Christus vor dem 50. Jahre gestorben ist, er also dann das höhere Alter gar nicht erreicht haben würde. Es bleibt also nichts anderes übrig, als anzunehmen, dass irgend ein Schreiber oder Leser, der sich in dem verwickelten Räsonnement nicht ausfand, in der Meinung es müsse der Endtermin des Lebens Jesu hier angegeben werden, den Zusatz aut oder et quinquagesimo gemacht hat.

I Semet ipsos obliti sunt solventes eius omne negotium et magis necessariam et magis honorabilem aetatem eius auferentes illam, inquam, provectiorem, in qua docens praeerat universis $(22,5)$ - Die Handschriften schwanken zwischen et docens, edocens und docens. 
Wie aber kommt Irenaeus dazu, das 40. Jahr als Grenzscheide der Jugend und des Alters hervorzuheben? Seine Argumentation verlangt, dass Jesu Lehrthätigkeit in das höhere Alter fält, sie verlangt nicht, dass diese eine längere Dauer hatte, oder, wenn er sich an das vierte Evangelium halten wollte, doch nur eine dreijährige. Die ganze Schwäche der Beweisführung aber wird gerade dadurch aufgedeckt, dass das vierzigste Jahr in den Kreis der Erörterung gezogen wird. Denn da die übereinstimmende Angabe der vier Evangelien, dass Jesu Lehrthätigkeit gleich nach der Taufe begonnen habe, stillschweigend anerkannt wird, so folgt, dass diese Lehrthätigkeit zum grösseren Teil in das Jugendalter fiel, was doch gerade widerlegt werden soll.

Es ist derselbe Widerspruch, den wir oben bemerkt haben. Vergebens sucht sich Irenaeus um Lc 3, 23 herumzudrücken. So sehr er sich auch dreht und windet, so kommt er doch an diesem Steine nicht vorbei. Sein ganzes Räsonnement bekommt erst Sinn und Zusammenhang, wenn das vierzigste Lebensjahr als Anfang der Lehrzeit Jesu gesetzt wird. Dann versteht man, warum von diesem Jahre an das höhere Alter gerechnet wird und dann erst kann man sagen, seine Lehrzeit sei in dieses Alter gefallen.

Diese Voraussetzung aber finden wir in der Notiz des Anonymus im wesentlichen erfüllt. Nach ihr kommt Jesus in einem Alter von 37 Jahren zur Taufe und lehrt bís zum 49. Das entspricht zwar nicht genau den Zahlen, aber doch der Anschauung, die der Argumentation des Irenaeus zu Grunde liegt. Die Differenz der Zahlen aber erklärt sich leicht, weil die Reçhnung imit runden Zahlen für Irenaeus unauffälliger war, wenn er das Anfangsjahr der Lehrzeit Jesu vertuschen wollte.

Wenn Papias und seine Presbyter Jesu Tod in sein 49. Lebensjahr setzten, so entspricht diese Schätzung dem vierten Evangelium so gut, dass man kaum umhin kann, sie aus ihm abzuleiten. Allerdings bleibt dabei völlig unerklärt, wie sie dazu kamen, eine zwölfjährige Lehrthätigkeit Jesu anzunehmen. Man kommt unwillkürlich auf den Gedanken, dass dabei eine Zahlenspielerei zu Grunde liegt und sie das Leben Jesu, das sie auf 48 volle Jahre ansetzten, in vier Abschnitte zu je 12 Jahren zerlegten. Denn dass sie von dem zwölfjährigen Jesus im Tempel, wenn auch nicht aus dem Lucasevangelium, gehört hatten, würde mir nicht unwahrscheinlich sein. Doch das ist eine blosse Vermutung, der ich keine weitere Stützen zu geben vermöchte. Dagegen scheint mir die Abhängigkeit des Papias vom vierten Evangelium gesichert. 
Muss es nun für sehr wahrscheinlich, ja beinah gewiss gelten, dass Papias das vicrte Ėvangelium gekannt hat, so findet sich dagegen in keinem der auf ihn mit einiger Sicherheit zurückgeführten Fragmente eine Andeutung über die Entstchung des Evangeliums. Es fragt sich, ob sich beweisen lässt, dass davon in seinem Werke überhaupt nichts gestanden haben kann.

Zunäclist haben wir festzustellen, dass, wenn Papias das vierte Evangelium kannte und benutzte, eine Bemerkung über seine Entstehung durch die Natur seines Werkes nicht nur nicht ausgeschlossen, sondern sogar nahe gelegt war. Hat er doch auch die Überlieferungen des Presbyters Johannes über das Marcus- und Matthäusevangelium mitgeteilt und behauptet er doch, mit Männern in Verbindung gestanden zu haben, die, wenn irgend wer, Träger einer solchen Uberlieferung über das Johannesevangelium hätten sein müssen, wenn anders es eine solche gab.

Von den beiden sorgfältigsten Lesern des Papias, die wir kennen, Eusebius und Irenaeus, verrät keiner, dass er bei ihm eine solche Überlieferung gefunden habe. Man hat gesagt, das Schweigen des Eusebius beweise nichts, da er für Schriften, die ihm als zweifellos galten, keine Zeugnisse sammelte. ${ }^{x}$ Aber so liegt die Sache doch nicht. Es handelt sich hier gar nicht um ein Zeugnis für die Echtheit des Evangeliums, sondern für die Umstände seiner Entstehung, die für Eusebius genau so interessant hättẹn sein müssen, wie der Bericht des Presbyters Johannes über Matthäus und Marcus. Da er uns diesen mitteilt, so wäre es nicht recht $z u$ begreifenn, warum er uns einen über Johannes hätte unterschlagen sollen. Noch schwerer wiegt das Schweigen des Irenaeus. $\mathrm{Er}$, der die Überlieferungen der Männer, die mit dem Apostel verkehrt haben wollten, so hoch schätzte, würde es doch sicher nicht verschwiegen haben, wenn er bei Papias etwas über die Entstehung des Evangeliums gelesen hätte, um so weniger, als er von denen wusste, die behaupteten, das Evangelium köönne nicht von dem Apostel geschrieben sein (III, II, 9).

$\mathrm{Zu}$ einem zwingenden Argument aber wird das Schweigen beider, wenn wir ihre Zeugnisse mit einander combinieren. Mit äusserster Kürze spricht sich Iren. III, I, I über die vier Evangelisten aus. Doch weiss er von Matthäus und Marcus Nebenumstände, von Lucas und Johannes sagt er nur aus, dass der eine Begleiter des Paulus, der andere der Lieblingsschüler des Herrn gewesen sei, der sein Evangelium in Ephesus verfasst habe, also nichts, was aus einer besonderen Überlieferung stammte.

I So Lightfoot, Contemp. Rev. Bd. 26, S. 854 cf. Bd. 25, S. 183 ff. Zahn, Gesch. d. Kanons, S. 9.00. 
Eusebius III, 39 berichtet von Marcus und Matthäus dasselbe wie Irenaeus, er schweigt von Lucas und Johannes. Beide schöpfen also aus derselben Quelle, Eusebius aber nennt Papias ausdrücklich als seinen Gewährsmann. Folglich hat Papias weder von der Abfassung des Lucas- noch des Johannesevangeliums etwas zu erzählen gewusst. ${ }^{2}$

Es giebt allerdings eine anonyme Notiz in einer lateinischen Evangelienhandschrift des 9. Jahrhunderts, nach welcher Papias in seinem Werke berichtet haben soll, er habe das Evangelium des Johannes nach seinem Dictat niedergeschrieben. Die Notiz lautet folgendermassen:

Euangelium Johannis manifestatum et datum est ecclesiis ab Johanne adhuc in corpore constituto, sicut Papias nomine Hierapolitanus, discipulus Johannis carus, in exotericis id est in extremis quinque libris retulit. Descripsit vero euangelium dictante Johanne recte. Verum Marcion haereticus, cum ab eo fuisset improbatus, eo quod contraria sentiebat, abiectus est a Johanne. Is vero scripta vel epistolas ad eum pertulerat a fratribus, qui in Ponto fuerunt. ${ }^{2}$

Von diesem Zeugnis machen Lightfoot (Cont. Rev. Bd. 26 p.-85 I ff.) sowohl wie Zahn Gebrauch, obwohl sie seinen höchst verdächtigen $\mathrm{Ge}$ samteindruck nicht in Abrede stellen. Mit Recht weist Lightfoot darauf hin, dass in einem Ausdruck, wie adluuc in corpore constituto das griechische Original noch durchschimmert, und ansprechend erklärt er exotericis für eine Verderbnis aus exegeticis, das einmal so verderbt dann mit extremis erklärt worden sei. Da mit der Notiz im ganzen nichts anzufangen ist, so hat Zahn die letzte Hälfte von Descripsit vero an über Bord geworfen und so hat auch Harnack und nach ihm Preuschen den ersten Teil unter die Fragmente des Papias aufgenommen: Aber was berechtigt uns zu sagen, die Angabe, dass Papias das Evangelium niedergeschrieben, erhebe nicht den geringsten Anspruch darauf aus dem Buche des.Papias geschöpft zu sein? (Zahn S. 899 Anm. I.) Ist doch dies das einzig interessante an der ganzen Notiz. Denn was bleibt ausserdem übrig, was, die Autorschaft des Johannes vorausgesetzt, nicht für jedermann selbstverständlich wäre, es sei-denn, er wäre ein Spiritist? Lightfoot und Harnack (Chronol. S. 665) suchen sich durch Conjecturen

× Vgl. meine Monarchianischen Prologe S. III. - Es verdient bemerkt zu werden, dass selbst Zahn sich dieser Einsicht nicht verschlossen hat. („Das einzige, was wir mit einiger Sicherheit daraus [d. h. dem Schweigen des Eusebius] schliessen dürfen, ist dies, dass Papias keine solche Erzählung über die Ursprungsverhältnisse oder die Ver. anlassung des 3 . und des 4 . Ev. gegeben hat." S. 897 a. a. O.)

2. Zahn, Kanonsgesch. S. 898 Anm. und meine Monarch. Prol. S. 114. 
mit der Sache abzufinden, während es doch, wie mir scheint, nur zwei gangbare Wege giebt: entweder man glaubt, dass Papias geschrieben habe, was dic Notiz behauptet, oder man glaubt es nicht. In dem ersteren Falle setzt man sich mit Irenaeus und mit Eusebius in Widerspruch und mit allem, was sich sonst aus cincr sorgfältigen Erwägung der vorhandenen Factoren ergicbt. Im zweiten Falle, beurteilt man dic erste Hälfte, wie man die zweite olhnehin beurteilt. Das scheint mir consequenter. Wie der Irrtum entstanden ist, wird nicht sicher zu sagen sein, aber sehr wahrscheinlich scheint mir, dass eine Verwechselung von Papias und Prochorus stattgefunden hat, dem Verfasser von Acten des Johannes, welcher genau das von sich behauptet, was hier dem Papias zugeschrieben wird."

Ich glaube, dass durch die bisherigen Erörterungen die Thatsachen bis zu demjenigen Grade der Wahrscheinlichkeit festgestellt sind, der in solchen Dingen zu erreichen ist. Wir können nun dazu übergehen aus den Thatsachen Schlüsse zu ziehen.

Wenn Papias und seine Gewährsleute das vierte Evangelium gekannt, doch aber nichts von seiner Entstehung zu berichten wussten, was folgt daraus für ihr Verhältnis zu dem Apostel Johannes, was für das Verhältnis dieses zu dem Evangelium?

Wenn wir uns erinnern, dass Irenaeus das, was er von den Presbytern zu erzählen weiss, aus Papias geschöpft hat, dass Papias dies nicht von den Presbytern selbst erfahren hatte, sondern von solchen, die jenen gefolgt sein wollten, dass Papias weder die Namen dieser seiner Gewährsleute noch auch der Presbyter genannt hatte, dass er endlich aus der Erinnerung und höchst wahrscheinlich erst in vorgeschrittenen Jahren schrieb - so werden wir gestehen müssen, dass wir einer solchen Überlieferung von vornherein viel Vertrauen entgegenzubringen nicht be-

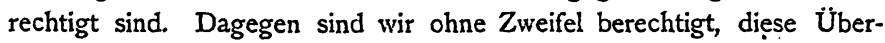
lieferung nach ihrem inneren Werte abzuschätzen.

Wenn nun die Gewährsleute des Papias behaupteten, der Apostel Johannes habe den Presbytern erzählt, dass ein tausendjähriges Reich anbrechen werde, in welchem die Weinberge und Felder für die Gläubigen millionenfache Frucht tragen würden, so werden wir, ohne Widerspruch befürchten $\mathrm{zu} \cdot$ müssen, die folgende Alternative aufstellen dürfen: wenn der Apostel Johannes das erzählt hat, so ist er nicht der Verfasser des vierten Evangeliums; wenn er aber der Verfasser des vierten Evangeliums

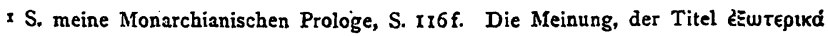
liesse sich als Nebentitel des papianischen Werkes rechtfertigen (S. 115), erledigt sich durch das oben S. 212 f. Gesagte. 
ist, so hat er das nicht erzählt. Wenn also die Presbyter das vierte Evangelium citiert haben, so haben sie entweder gewusst, dass dies Evangelium nicht von Johannes war, oder sie haben zu Unrecht behauptet, mit dem Apostel verkehrt zu haben.

Kein Apostel kann erzählt haben, Jesus sei in einem Alter von 49 Jahren unter Nero gekreuzigt worden. Will man aber doch annehmen, es sei einer von ihnen so alt und altersschwach geworden, dass er die Zeiten,nicht mehr habe unterscheiden können, so wird diese angebliche Behauptung der Presbyter dadurch aufs äusserste discreditiert, dass einige von ihnen sich dafür nicht nur auf Johannes, sondern auch auf andere Apostel berufen haben sollen.

Wäre es unrecht, Leute, die solche Dinge erzählten, betrogene Betrüger zu nennen? Jülicher, dem ich für die Ausstellungen, welche er einst an meinen Monarchianischen Prologen gemacht hat, dankbar bin, weil sie zumeist mich $\mathrm{zu}$ einer erneuten Prüfung meiner Ansichten an-

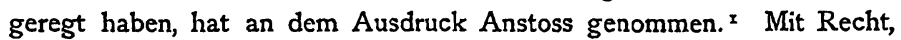
sofern er auf die Presbyter angewendet war. Was wissen wir von diesen Presbytern, was wissen wir, ob jemals Presbyter solche Behauptungen gewagt haben? Der einzige, den wir verantwortlich machen können und müssen, ist Papias. Ihn aber können wir von dem Vorwurf bodenloser Leichtgläubigkeit und Kritiklosigkeit nicht freisprechen. Wer eigentlich hinter ihm steht, können wir nicht mehr ausmachen, da er die Gewährsmänner, die uns am meisten interessieren, nicht nennt und die, die er nennt, der Presbyter Johannes und Aristion², für uns blosse Namen sind. Aber wir sehen doch, dass Papias keineswegs allein stand, sondern dass die Überzeugung, es habe noch im 2. Jahrhundert Zeugen der Geschichte des Herrn gegeben, weiter verbreitet war. So erzählte Hegesippus, dass Symeon, der Vetter des Herrn, unter Trajan gekreuzigt sei, nachdem er in einem Alter von 120 Jahren eine vieltägige Folter ausgehalten habe. (Eus. h. e. III, 32, 3. 6.) Von den von Christus erweckten Toten aber, die bis Hadrian gelebt haben sollen, stand auch in der Apologie des Quadratus zu lesen (h. e. IV, 3, 2). 3

2 Gött. gel. Anz. 1896, S. 841 ff.

2 Harnack identificiert nach Rohrbach mit Aristion den in einer armenischen Evangelienhandschrift als Verfasser des unechten Schlusses des Marcusevangeliums genannten Presbyter Ariston (Chronol. S. 697 f.), Resch dagegen denselben mit Aristo von Pella, dem Verfasser des Dialogs des Jason und Papiscus, wogegen Harnack seinerseits protestiert (Chronol. S. 269).

3 Ich möchte daraus nicht mit Harnack (Chronol.S. 270) schliessen, dass Papias von Quadratus abhängig sei. Die Sache verlangt aber eine längere Auseinandersetzung, 
Dies zeigt, dass die mündliche Tradition der kirchlichen Kreise auf dem Wege völliger Entartung war. Es war höchste Zeit, sie ein für allemal abzuschliessen; die Kirche hat dies Bedürnis gefühlt und hat ihm Genüge gethan, indcm sic den Kanon geschaffen hat. Yapias steht nitten in diesem Processe drin, und insofern er dazu beiträgt, ihn zu verstchen, ist er eine interessante und beachtenswerte Erscheinung. Die schriftlichen Evangelien waren da, aber man glaubte ihren Stoff noch aus der mündlichen Überlieferung vermehren zu können, wie die angeblich von dem Apostel Johannes berichtete Äusserung Jesu uber die $\mathrm{Zu}$ stände des tausendjährigen Reiches zeigt. Die Evangelien galten noch nicht als die ausschliessliche Erkenntnisquelle der apostolischen Überlieferung über die Aussprüche und das Leben des Herrn, aus dem einfachen Gruncle, weil ihre Zahl noch nicht abgeschlossen war. Denn offenbar bezeugt Papias so gut wie Lucas eine Vielheit von Evangelien. Anders lässt sich die Bemerkung nicht verstehen, dass es eine Menge von Übersetzungen des hebräischen Matthäusevangeliums gegeben habe. Bemerkenswert aber ist dabei, das diese vielen Evangelien auf einen $\mathrm{Ur}_{\mathrm{r}}$ sprung zurückgeführt werden und dass aus der Menge ein Evangelium des Marcus ausgesondert wird. Wenn man aber annahm, dass es von Matthäus viele Übersetzungen gab, so wird man auch den Versuch gemacht haben, darunter eine auszuwählen, die als die bestbeglaubigte gelten konnte. Es ist durchaus wahrscheinlich, dass das bereits $\mathrm{zu}$ Papias' Zeit geschehen war. Wir haben ferner gesehen, wie alles dafür sprach, dass schon Papias. Matthäus und Johannes als diejenigen Apostel ansah, die Evangelien verfasst hätten. Das alles zeigt, wie weit die Kanonbildung schon vorgeschritten war. Nur die lukanischen Schriften hatten sich offenbar noch keine Anerkennung errungen.

Kehren wir nun $z u$ der Frage zurück, von der wir ausgegangen sind, so hat sich mit höchster Wahrscheinlichkeit ergeben, dass Papias das vierte Evangelium gekannt und benutzt hat. Hat er das aber gethan, so muss er es für mittelbar oder unmittelbar apostolisch gehalten haben. $\mathrm{Da}$ nun nach ihm der Glaube an den johanneischen Ursprung des Evangeliums in kirchlichen wie in häretischen Kreisen gleich weit verbreitet ist und da auch seine Bestreiter annahmen, dass das Evangelium auf den Namen des Johannes geschrieben sei, so kann Papias nicht wohl

die sich von dem vorliegenden Gegenstande zu weit entfernen würde. [Vgl. dazu den in einem der nächstèn Hefte der Zeitschrift erscheinenden Aufsatz des Verf. „Die Töchter des Philippus". E. P.] 
einen anderen für den Verfasser gehalten haben. Es hat sich aber zugleich herausgestellt, dass Papias so wenig wie die Folgezeit ein äusseres Zeugnis dafür besessen hat. Besass aber Papias ein solches Zeugnis nicht, so werden wir mit einiger Sicherheit behaupten dürfen, dass das christliche Altertum überhaupt keines besessen hat. Gab es aber kein äusseres Zeugnis, so kann die Úberzeugung, dass das Evangelium von dem Apostel Johannes verfasst sei, sich nur auf das Selbstzeugnis des Evangejiums gegründet haben.

Warum aber wurde dieses auf den Apostel Johannes gedeutet? 